\title{
Patrón adaptativo obesogénico cerebral: una propuesta para comprender y evaluar la obesidad en la práctica clínica
}

\author{
Adaptative obesogenic brain pattern: \\ a proposal for understanding and \\ assessing obesity in clinical practice
}

\begin{abstract}
Obesity appears to be a complex condition. Current treatments are based on simple models of input and output power with regular short-term results. It is possible that changing the model of understanding long-term, better results are achieved. Neurobiological research proposes to consider the existence of an Adaptative Obesogenic Pattern (AOP) where the functioning of the nervous system is organized to increase intake. AOP is proposed as an early and maladaptive response establishing that the reward systems can modulate the axis HPA (hypothalamic pituitary adrenal axis) in the absence of sufficient social regulation. This would facilitate the reverberation of the reward system by exposing it to addictions. Based on the failure of integration between axis HPA a model is presented. Child and adolescent observational grid is proposed to evaluate AOP during the current clinical interview. Key words: obesity; stress; addiction; regulation; weight.
\end{abstract}

Patricia Cordella M. (1)

Catalina Moore I. (2)

(1) Unidad de Familia y Salud. Programa de Trastornos de Alimentación. Departamento de Psiquiatría Pontificia Universidad Católica de Chile. Santiago, Chile. (2) Unidad de Trastornos de Alimentación Departamento de Psiquiatría. Pontificia Universidad Católica de Chile, Santiago, Chile

Dirigir la correspondencia a: Dra.

Patricia Cordella M Fono 22205455

E-mail: mpcordella@gmail.com

Este trabajo fue recibido el 27 de Julio de 2014, aceptado con modificaciones el 1 de Septiembre de 2014 y aceptado para ser publicado el 10 de Noviembre de 2014.

\section{INTRODUCCIÓN}

La obesidad es una condición de riesgo y alto costo económico que preocupa a los países por su proporción endémica. Se estima que $16.9 \%$ de niños y adolescentes entre los 2 y 19 años (USA) (1), y más de 40 millones de niños menores de 5 años estaban con sobrepeso u obesidad el año 2012 (2).

En Chile según cifras del Minsal, la prevalencia de sobrepeso escolar ha ido en rápido aumento los últimos 4 años, llegando al 20\%. La obesidad en menores de 5 años alcanza más de $10 \%$ (3), y en los mayores alcanzan una cifra de $23.2 \%(4,5)$.

Los estudios realizados en el seguimiento de pacientes en tratamiento muestran regulares resultados a corto plazo (6). A largo plazo aparecen los rebotes de peso como si existiera una tendencia a volver a un punto de equilibrio que se sostiene con una alta ingesta y un incremento del IMC (índice de masa corporal). Las teorías del set point glucostático (7) y lipídico (8) no fueron completamente convincentes para explicarlo, aunque propusieron dos premisas importantes para la comprensión de la obesidad: algo ocurre a nivel del sistema nervioso central que tiende a repetirse y lo que sea, es resistente al cambio.

Desde el inicio el SNC se ocupa de regular el estrés y así aumenta las posibilidades de supervivencia. El sistema de apego lo logra regulando la distancia entre los cuerpos siendo aún más básico y eficiente entre los mamíferos el sistema de ingesta.

La ingesta genera un pick de dopamina asociado al el triángulo límbico (9) formado por el HMV (hipotálamo Medio Ventral) el NA (Núcleo Acumbens) junto al TA (Área tegmental) y la amígdala que logra disminuir la alerta. Sin embargo si el estrés solo estuviera regulado a través de estas conexiones cerebrales centrales, la obesidad sería una regla general (10-12).

El principal sistema implicado en el estrés crónico es el eje HPA (hipotálamo-Hipófisis-Adrenal) el cual con múltiples aferencias hipotalámicas va aprendiendo a ajustar el nivel óptimo de cortisol según las necesidades adaptativas de ese individuo en ese contexto.

El aprendizaje/ andamiaje de los procesos regulatorios del estrés obedece probablemente a la repetición de formas fisiológicas socialmente adquiridas a través de acoplamientos repetitivos con los significativos afectivos. Estos patrones se consolidan en el tejido cerebral utilizando los mecanismos de aprendizaje LTP (Long Term Potentation) (13). Se deduce por lo mismo la importancia que tienen los cuidadores primarios en los primeros estadíos del desarrollo cerebral y psíquico como organizadores del tejido cerebral.

Dada la asociación que existe entre comer y ser cuidado en los inicios de la vida, es posible postular que el uso de la recompensa de la ingesta como regulador del estrés se esta- 
blezca como un patrón neuronal muy básico y temprano del tipo estímulo/ respuesta. Una unidad funcional elemental, la cual al quedar des- integrada de los desarrollos posteriores derivados del cerebro social como regulador del estrés someta a riesgo de obesidad a ese organismo.

Podríamos postular que si en el desarrollo temprano de los sistemas regulatorios al stress no se logra la integración entre los circuitos de recompensa quedando la ingesta como una de las tantas alternativas posibles, el resultado será un patrón adaptativo al estrés precario y primitivo que somete al organismo a repetir la conducta de búsqueda, ingesta y almacenaje de energía como solución indiferenciada y preferente al estrés.

Entonces ¿Cómo ocurriría la organización del acontecer cerebral de un modo eficiente en términos de balance energético para el organismo?

\section{LA MODULACIÓN DEL ESTRÉS EN EL DESARROLLO NORMAL}

Al nacer cada niño parece venir dotado de un grado de adaptación al medio que llamamos temperamento el cual pone a prueba las habilidades de sus cuidadores para mantenerlo en bienestar (14).

Los procedimientos de aquietamiento se encuentran posiblemente precableados en los mamíferos (15) configurando los llamados circuitos primarios de filiación o apego. Se despliegan en las conductas de reunión y separación entre los cuerpos mamíferos posibilitando el cuidado a través de un sistema neuro-afectivo-conductual. Es ya conocido que los patrones de apego seguro logran mejor su objetivo que los inseguros o caóticos (16).

Durante el primer año de vida, el cuerpo de la madre es el principal regulador del estrés (17) a través del abrazo y el alimento. Ingerir y calmar se viven juntos en esta etapa y se habla de simbiosis madre-hijo aludiendo a la indiferenciación entre funciones. Será a través del nacimiento de un individuo mentalmente separado de sus cuidadores, que se logrará escindir el pack "ingesta-recompensa-calma" hasta alcanzar la calma psíquicamente lograda. Este proceso requerirá tanto de mecanismos de mentalización (18) como de incorporación de los modos culturales de significar la fisiología del organismo humano (19).

\section{Ingesta y estrés}

La baja de glucosa intracelular inducida por estrés activaría también el aumento de ingesta a través de aumentar las hormonas orexígenas (20), vía circuitos mesolímbicos de recompensa y vía ACTH (hormona corticoestimulante). Esta última liberada por el estímulo de CRF (factor liberador de corticotrofina), vía amígdala y otras células receptoras de alarma dispuestas por todo el tejido cerebral.

Una disminución de la glucosa cerebral ha sido encontrada en obesos, diabéticos y enfermos con depresión (21), mostrando cómo este mecanismo pudiera estar ligado a las tres situaciones.

Bajo condiciones normales, el aumento de la glucosa intracelular inhibiría las conductas gatilladas por HPA, pero bajo estrés crónico la respuesta se alarga y más zonas son activadas, aumentado de este modo el número de células que producen Factores Liberadores de Corticotropina (CRF). Por otra parte, La hormona corticotropina (CRH), induce la potenciación de larga duración (LTP) en la zona ventrotegmental (VTA) y la amígdala facilitando el aprendizaje, es decir, la formación de dendritas que asociarían el sistema de estrés con el de recompensa/ comer bajo situaciones de estrés. Esto ocurre especialmente si la recompensa es positiva, ya sea por su palatibilidad o significado. El refuerzo será entonces positivo y reverberarán los circuitos produciendo un aprendizaje condicionado que facilita y consolida el impulso a comer.

A pesar que las hormonas orexígenas son producidas exclusivamente por el hipotálamo, sus axones y receptores están ampliamente distribuidos en el cerebro. Si inyectamos hormonas orexígenas, se produce una respuesta fisiológica similar al stress (22) llegando a producir hasta pánico y bloqueo en ratas (20), lo que indica la cercanía fisiológica y anatómica que tienen estos circuitos. Las hormonas orexígenas están conectadas con el sistema de estrés y también con el sistema de recompensa que se activaría con la ingesta (23).

\section{Recompensa y estrés}

El hipotálamo integra señales relacionadas con los procesos de motivación a la recompensa venidas tanto del tronco cerebral, la corteza y el sistema límbico, y allí están las hormonas orexígenas ligando motivación y comida. El sistema de recompensa incluye: Hipotálamo Lateral produciendo hormonas orexígenas, Área Ventro Tegmental (VTA) y Núcleo Accumbens (NA). El VTA tiene muchos receptores de hormonas orexígenas, que a su vez aumentan la dopamina incluso en la corteza prefrontal (CPF) (24). La activación frecuente del sistema dopaminérgico de recompensa lleva a un proceso adaptativo donde se necesita cada vez más estímulo para encontrar la misma respuesta, provocando con esto un patrón de adicción.

El eje HPA crónicamente activado incrementa la ingesta de alimentos, especialmente de aquellos que generan recompensa vía dopamina como son los carbohidratos y los alimentos ricos en grasas, ya que suelen tener mayor palatabilidad (11). El estrés por si solo estimula al sistema de recompensa vía amígdala/ VTA y a través de las hormonas orexígenas, así como el de opioides endógenos activando todo como un mecanismo de defensa neurofisiológico que hace posible sobrevivir al estrés (25,). Como la ingesta es la forma más accesible y poderosa de reducir la actividad del eje HPA, comer es una solución eficiente que puede ser usada sin necesidad de realizar los procesos psíquicos más complejos que requieren la maduración de las áreas involucradas en la simbolización de la experiencia. Podemos decir que la ingesta no requiere de mente, es primitiva, innata como sistema de regulación.

La obesidad infantil temprana sería la expresión en grasa de la activación crónica de estos circuitos en ausencia de otro humano que lo haya modulado eficientemente. Este patrón es similar aunque no idéntico al que muestran los enfermos de trastorno por atracón o los adictos a grasa y azúcares (26).

La intervención terapéutica en el eje HPA requeriría subir el nivel de funcionamiento hacia uno de mayor complejidad, es decir, establecer conexiones con áreas de simbolización ubicadas en la corteza cerebral asociados a la simbolización. Para esto es necesario ligar diferentes áreas del tejido nervioso de modo de complejizar, ampliar y dar particularidad a las respuestas adaptativas al estrés que requieren de la interacción con un ser humano que lo haya logrado (adulto) de modo de copiar por mimesis o vía neuronas espejo (27-29) el andamiaje estructural sin que reverbere en los circuitos de recompensa.

Apego y estrés

Son múltiples las fuentes de estrés a las cuales un bebé se ve expuesto: ruidos, cuerpos que simulan depredadores, olores desagradables, sensaciones táctiles además de la presencia/ 
ausencia de su entorno social. Por otra parte, sus cuidadores también pueden ejercer su función de protección del ambiente de modo inconveniente para su bienestar, gatillando los mecanismos de alarma.

La alarma es particularmente sensible a la distancia de los cuerpos cuidadores y se enciende ya sea por el abandono o la intrusión desafiando al niño a autoregularse.

Regularse por sí mismo significa replicar el estado fisiológico de bienestar logrado con el cuidador. Cuando el niño, explora impulsado por su propia motivación, se distancia de la madre y arriesga sentir angustia. Sin embargo, si la motivación por aquello que está allá es alta, la angustia en vez de bloquearlo lo impulsará a la acción. Fuera del campo de vigilancia y cuidados de sus significativos emocionales, deberá activar sus propios circuitos de regulación que se irían estableciendo en zonas del hipocampo relacionadas con la memoria procedural (30) creadas por él a partir de las interacciones cotidianas que activan las neuronas espejo $(28,29)$.

El sistema de autorregulación se establecerá entorno a los cuatro años (31). Sin embargo, si los cuidados parentales no han sido suficientes y la regulación falla tempranamente es posible que haya aprendido a regular el eje HPA a través del sistema de recompensa asociado a la ingesta y que ya sea un niño obeso.

Regulación social del estrés: mentalización

La mentalización, como su nombre lo indica, es la construcción a lo largo del desarrollo del llamado aparato metal. Una serie de procedimientos dispuestos para regular una fisiología acoplada al ambiente de un modo saludable. La mentalización es el proceso a través del cual los cuidadores traspasan al niño los procedimientos de autogestión fisiológi$\mathrm{ca}$, emocional y afectiva de su cultura (32). Dos condiciones propias de los mamíferos deben darse para hacer el traspaso de este saber acumulado. Por una parte la empatía (33), y por otra la llamada Teoría de la Mente $(34,35)$.

a) Por empatía entendemos la capacidad de sintonizar con la experiencia emocional de otro cercano, para así comprender su estado emocional de un modo pre verbal. Una vez aprehendida, se requiere la capacidad de interpretarla y devolverla transformada en algún código simbólico (movimiento, palabra, diagrama).

b) La Teoría de la Mente surge de los estudios con primates no humanos y luego con niños pequeños, y consiste en la capacidad de inferir el estado mental y la conducta futura de otro ser vivo leyendo sus motivaciones, pensamientos e intenciones antes que las realice de modo de ajustar en el aquí -ahora la próxima acción.

Estas dos capacidades innatas de la especie se apoyan para funcionar en el sistema de neuronas espejo (36), y especialmente en la lectura de la cara (37). Nuestra especie se caracteriza por ser capaz de manejar diversos códigos (acuerdos) simbólicos que complejizan la administración fisiológica del cuerpo al anexarle significados culturales. Es decir, podemos regular las respuestas fisiológicas a través de procesarlas en los filtros de significados positivos o negativos que la cultura les tiene preferentemente asignados.

La relación que establece el niño con su madre facilita un campo intersubjetivo desde el cual emergen los procesos de sintonización, sincronización (38) y ampliación del estado de conciencia $(39,40)$ entre otros, todos los cuales se basan en la empatía y la teoría de la mente.

Es a través de estos procesos que se traspasan los modos de gestionar estados emocionales, especialmente los relaciona- dos con la alarma ambiental que justamente usan el eje HPA El sistema de alarma está representado por la amígdala, el sistema CRF distribuido en el cerebro, el hipocampo y la CPF los cuales van siendo moldeados por los cuidadores. Ellos realizan la tarea de mentalizar, es decir, de ir uniendo los estados fisiológicos del niño con algún referente que le dé significado $(41,42)$. Los cuidadores proponen también el tono de alarma basal, la línea base del funcionamiento y desarrollo de los potenciales del Sistema Nervioso. Esta línea participaría en la distribución y organización ponderada de grandes porciones del cerebro (43). Esta basal es medible a través de diversos tests (44) que observan y puntúan conductas productoras de salud mental en adultos (45).

Cuando hablamos de relación diádica nos referimos al ambiente social y emocional del desarrollo cerebral-mental. Es el lugar de encuentro desde el cual será regulado el estrés a través de conductas de cuidado $y$, por otro lado, donde se produce la copia y el acoplamiento a estos procesos (46). Es desde donde emergen los patrones de conexión regulatoria del estrés y la motivación. También es el lugar donde se produce la evaluación social y como tal puede ser fuente de estrés. Se está estudiando ampliamente de qué forma los pacientes con trastornos de alimentación conforman esta función social de la mente (47).

Por lo tanto, si pensamos en la modulación de estrés vía recompensa por alimentos como un mecanismo simple y básico que se salta la mentalización para autorregular el bienestar del niño, podremos entender que se llega a la obesidad desde un retraso en el desarrollo de la autonomía regulatoria del estrés. Esta alteración utiliza el sistema de recompensa para evitar el colapso del sistema HPA. Un HPA desregulado presentaría los síntomas característicos de angustia en sus formas de crisis de pánico, angustia generalizada, fobias entre otras, incluso algunos podrían llegar a fatigar el sistema de alarma y presentar depresión (48). El niño que ha tenido que regular tempranamente su eje HPA sin las habilidades mentales necesarias podría hacer uso frecuente de circuitos de recompensa a través de la ingesta y acumularía depósitos energéticos presentando una historia de obesidad infantil temprana secundaria al uso de un patrón adaptativo obesogénico. Este patrón no se establecería de igual forma si esta obesidad se inicia en etapas posteriores secundaria un trauma, una patología, el uso de un medicamento o malos hábitos alimenticios.

\section{La propuesta de un modelo:}

Patrón Adaptativo Obesogénico (PAO)

El PAO sería un patrón de respuesta al estrés temprano que utiliza el sistema de recompensa como modulador del eje HPA, saltándose la modulación relacional y/o la mentalización de los estados de disconfort. El PAO estaría conformado por tanto por alteraciones en el sistema de regulación del estrés, la recompensa y motivación (49), y en las funciones del cerebro social.

EI PAO sería una vía neurofisiológica rápida capaz de regular el eje HPA. Evolutivamente hablando corresponde a una solución de mamífero, es decir a una solución sin mente, desmentalizada, primitiva y precaria para el manejo especialmente del estrés social al que somos expuestos en el acontecer relacional cotidiano (50).

EI PAO presentaría fallas en la integración de la experiencia que derivarían en momentos de disociación $(51,52)$ y malestar irracional impulsando el consumo de alimentos como forma primaria de resolución. 
El principal efecto colateral del PAO es el aumento de peso y con éste una figura fuera de la norma aceptada socialmente, vivida con vergüenza por el niño. En ocasiones esto lo lleva a aislarse o a luchar por pertenecer al grupo de pares, agregando estrés cerrando el círculo de malestar. Dependiendo de la disposición innata del individuo, puede deprimirse, desesperarse, enojarse o angustiarse, volviendo a buscar en la comida la solución al disconfort. Este tramo de la dificultad es lo que se ha llamado el comer emocional, es decir, buscar en el comer sin encontrar, la solución a una indefinida sensación de malestar.

EI PAO entonces estaría formado por la integración maladaptativa de los siguientes sistemas:

a) Regulación del eje del estrés (53); b) Motivación, recompensa y adicción al comer (54-56); c) Desarrollo social (57-60).

Podemos ver en la tabla 1 los sistemas implicados en el PAO $(9,61-64)$. En el desarrollo normal estos sistemas funcionan interconectados y sin necesidad de ser estimulados preferentemente por alimentos, en cambio en el PAO el aumento del estrés gatilla el de recompensa y elimina el componente social. En la figura 1 vemos cómo la regulación de la ingesta está conectada con la regulación relacional, quedando ambas ligadas al eje HPA y al sistema de recompensa. El otro ser humano también representa una fuente de motivación y placer, siendo uno de los puntos de salida del sistema que reverbera en el comer.

La obesidad se ha relacionado con diversas alteraciones del desarrollo del sistema nervioso ligadas a fallas como son: la alexitimia $(65,66)$; la falta de empatía (67); el apego inseguro (68) que remiten tanto al eje HPA, como al de recompensa. Comprender la obesidad como una patología cerebral ha sido ya descrito por varios autores $(69,70)$.

¿Cómo se puede reconocer cuales son las diadas en riesgo de PAO? Es decir ¿qué signos son capaces de mostrar que esa díada tiene un eje HPA desregulado y que dado que el hijo tiene obesidad es muy probable que se haya establecido un PAO? Un screening podría mostrar la pertinencia de intervenir.

Para que se haya establecido un PAO la historia del niño nos mostrará lo temprano que aparece el sobrepeso y la cronicidad de la ingesta como se ha hecho crónica la ingesta regulatoria así como las conductas de búsqueda y almacenamiento de alimentos.

A través de la psicoterapia estas fallas se pueden reparar

\section{TABLA 1}

Sistemas involucrados en el PAO (Patrón Adaptativo Obesogénico).

\begin{tabular}{|c|c|c|c|}
\hline Sistema & Algunas formas de estudio & Zonas cerebrales implicadas & Función \\
\hline $\begin{array}{l}\text { Motivación y } \\
\text { recompensa }\end{array}$ & $\begin{array}{l}\text { a) Modelos animales } \\
\text { b) Respuesta cerebral a estímulos } \\
\text { de alimentos en humanos }\end{array}$ & $\begin{array}{l}\text { Striatum, mesencéfalo; CF orbital, lateral y } \\
\text { medial; amígdala; corteza gustativa; hipocampo } \\
\text { (giro dentado); núcleo accumbens }(9,61) \text {. }\end{array}$ & $\begin{array}{l}\text { Recompensa } \\
\text { Impulso } \\
\text { Motivación } \\
\text { Deseo }\end{array}$ \\
\hline Estrés & $\begin{array}{l}\text { a) Modelos animales } \\
\text { b) Tareas estresantes en humanos } \\
\text { c) Medir cortisol; ocitocina; Pro- } \\
\text { lactina; Vasopresina }\end{array}$ & $\begin{array}{l}\text { Amígdala; hipocampo (giro dentado); tálamo; } \\
\text { hipotálamo; corteza prefrontal }(62,63) \text {. }\end{array}$ & Alarma/ defensa \\
\hline $\begin{array}{l}\text { Dimensión social } \\
\text { relacional }\end{array}$ & $\begin{array}{l}\text { a) Empatía } \\
\text { b) Teoría de la mente }\end{array}$ & $\begin{array}{l}\text { Cíngulo; CPF; amígdala; hipocampo } \\
\text { (giro dentado); ínsula (64). }\end{array}$ & $\begin{array}{l}\text { Filiación } \\
\text { Cuidado } \\
\text { Reproducción }\end{array}$ \\
\hline
\end{tabular}

FIGURA 1

Modelo de POP (Patrón Obsesogénico Predominante).

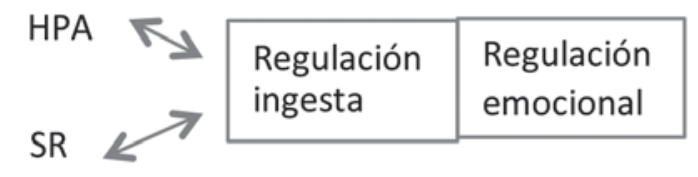

\section{Autonomía \\ Dependencia}

\footnotetext{
La figura 1 muestra como la regulación de la ingesta y la regulación emocional usan vías similares para activarse (eje HPA: hipotálamo-hipófisis-adrenal) y aquietarse (SR: Sistema de recompensa). La dimensión emocional se mueve entre los polos de la dependencia cuando requiere de la relación con otro para lograr la regulación y la independencia, cuando la puede realizar por sí mismo.

EI PAO (patrón adaptativo obesogénico) se saltaría los pasos intermedios (procesos de mentalización) ajustando la regulación emocional a través de la ingesta, lo que llevaría al frecuente aumento de peso.
} 
re-mentalizando a través de la comunicación social intencionada, un complejo sistema de reconocimiento y asignación de significado que guía la interacción (71).

\section{UNA FORMA DE EVALUAR EL PAO:}

\section{OBSERVANDO MIENTRAS SE TOMA LA HISTORIA CLÍNICA}

En la literatura existen numerosas formas de evaluar, las competencias sociales de un individuo (72), que abarcan los términos utilizados para evaluar la función social. Estos son: apego, comunicación, filiación, teoría de la mente y percepción facial $(73,74)$. También se ha utilizado el DOCS (Dyadic Observational Comminication skills) para evaluar díadas (75) a través de la observación de videos y consenso de observadores.

En la clínica sin embargo, es necesario utilizar algún instrumento sencillo y rápido que nos permita tomar decisiones sobre la marcha. Proponemos un screening relacional diádico (madre/hijo) basado en la observación que realiza el profesional mientras toma la historia del paciente. Puede ser utilizado para cualquier edad. Se trata de testear aquello que se observa de la comunicación verbal y no verbal entre la madre y su hijo, de modo de cuantificarla, obtener con esto un puntaje y hacer las categorías necesarias para la toma de decisión (tabla 2). Este tamizaje está basado en la observación clínica y recoge la tradición del Infant Research (76). Es una propuesta inédita que requerirá ser validada y reformulada por la práctica clínica y de investigación. El puntaje obtenido orienta el diagnóstico, pronóstico y las intervenciones.

Esto nos ayudará a identificar las diadas con riesgo, es decir con desregulación del eje HPA. Como este eje tiende a estimular el SS (sistema Simpático) y éste se encuentra en equilibrio con el SPS (sistema Parasimpático), podemos encontrar diadas complementarias o simétricas con respecto al funcionamiento del equilibrio SS/SPS. Son los extremos del funcionamiento los que muestran la desregularización del sistema de estrés.

El instrumento está compuesto por dos dimensiones: Apego y Teoría de la Mente. La primera tiene los indicadores de: a) Postura del niño; b) Postura de la madre; c) Coordinación diádica; d) Responsividad parental; e) Control del cuerpo; La segunda tiene los indicadores de: a) Representación del sí mismo del niño; b) Aceptación materna del niño) Negociación.

La postura es el lugar final de una serie de equilibrios que determinan el tono de alerta de un individuo. Este tono está básicamente regulado por un equilibrio del SNA (sistema nervioso autónomo), donde el sistema simpático aporta el alerta y el parasimpático su contraparte.

La línea propuesta va desde un organismo que está sobre compensado por el funcionamiento del parasimpático, hasta otro que está hegemonizado por el simpático. En el centro encontramos el mejor modo de funcionar. Así, el Derrumbado Pasivo, es un organismo que está desparramado en la silla y al que hay que atraer con alguna motivación al contacto. El Laxo es un cuerpo muy suelto, sin tono de alerta, que parece tener sueño y que le cuesta conectarse en la interacción con el evaluador. El Atento Tranquilo es un niño/ adolescente que estando presente, lo hace sin urgirse. Éste es el estado de aprendizaje, cuando un niño está conectado con los otros y atento a hacerle preguntas para comprender su ambiente. El Hiperalerta es un organismo muy despierto, que reconoce detalles de la interacción tanto de su madre como del entrevistador, que no deja pasar contradicciones, mira de frente y

\section{TABLA 2}

Definición de los puntos a evaluar en la regulación diádica.

\begin{tabular}{|c|c|c|c|}
\hline Ámbito & Dimensión & Característica & Signos \\
\hline \multirow[t]{4}{*}{$\begin{array}{l}\text { Sistema de } \\
\text { apego }\end{array}$} & $\begin{array}{l}\text { Postura corporal del niño } \\
\text { y de la madre }\end{array}$ & $\begin{array}{l}\text { Forma muscular de sostener el cuerpo } \\
\text { en reposo que refleja el tono de alerta. }\end{array}$ & $\begin{array}{l}\text { Posición de brazos piernas y manos } \\
\text { en relación al tronco }\end{array}$ \\
\hline & Coordinación diádica & $\begin{array}{l}\text { Ordenamiento de los movimientos } \\
\text { corporales diádicos a través de turnos } \\
\text { o acuerdos motores. }\end{array}$ & $\begin{array}{l}\text { Frecuencia de movimientos fluidos } \\
\text { conjuntos }\end{array}$ \\
\hline & Responsividad parental & $\begin{array}{l}\text { Capacidad del cuidador de darse } \\
\text { cuenta de las necesidades y estados } \\
\text { emocionales del niño, y asistirlo en la } \\
\text { resolución. }\end{array}$ & $\begin{array}{l}\text { Reconoce y actúa asistiendo } \\
\text { al niño }\end{array}$ \\
\hline & Control del cuerpo del niño & $\begin{array}{l}\text { Modo en el cual se realizan los } \\
\text { movimientos. }\end{array}$ & $\begin{array}{l}\text { Movimientos coordinados y } \\
\text { coherentes con el discurso }\end{array}$ \\
\hline \multirow[t]{3}{*}{$\begin{array}{l}\text { Teoría de la } \\
\text { mente }\end{array}$} & $\begin{array}{l}\text { Representación de sí mismo } \\
\text { del niño }\end{array}$ & $\begin{array}{l}\text { Idea de sí mismo que modula la } \\
\text { autoestima. }\end{array}$ & $\begin{array}{l}\text { Tipos de palabras y afectos asociados } \\
\text { a la descripción de sí mismo o de lo } \\
\text { que le ocurre }\end{array}$ \\
\hline & $\begin{array}{l}\text { Aceptación materna del } \\
\text { Niño }\end{array}$ & $\begin{array}{l}\text { Aprobación y recepción de las } \\
\text { características del otro }\end{array}$ & $\begin{array}{l}\text { Frecuencia de contacto ocular, } \\
\text { sonrisa, asentimientos. }\end{array}$ \\
\hline & Negociación & $\begin{array}{l}\text { Modo a través del cual se llega a } \\
\text { acuerdos. }\end{array}$ & $\begin{array}{l}\text { Frecuencia en la que se llega acuerdo } \\
\text { luego de ajustes corporales y verbales. }\end{array}$ \\
\hline
\end{tabular}


explora. El Amenazado Activo no sólo explora sino que desafía y desconfía del entrevistador. El Derrumbado Alerta dejó de estar atento al medio y parece estar en otro mundo, en esta modalidad se pueden encontrar estados psicóticos.

La misma dimensión en la madre puede aparecer con sutiles diferencias, aun representando lo mismo. La Confusa (ambivalente) es la que hace movimientos no congruentes con la situación, por ejemplo, habla por teléfono al entrar a la consulta y no corta, o abraza al hijo mientras lo critica. La Pasiva Tranquila espera que alguien haga algo, se mueve poco y espera que le digan lo que tiene que hacer, parece sumisa. La Alerta Tranquila está atenta a la interacción tanto del hijo como del entrevistador y reacciona con mesura. La Hiperalerta es rápida, mira de frente, se apoya en la punta de la silla, mueve al hijo con energía. La Defensiva Amenazada está en posición adelantada, mira fijamente a los ojos, tiene la musculatura tensa en todo el cuerpo. La Delirante está inquieta, gira la cabeza o el tronco, no sostiene la postura acoplada a lo que acontece.

La coordinación diádica se refiere a cómo los cuerpos de ambos van haciendo figuras coherentes que respetan turnos de acción, van acordando velocidad, tipos de movimientos, puntos de atención. Así la Disociada es una forma en que cada cuerpo realiza actividad sin tomar en cuenta al otro, aparecen por ejemplo, uno alerta y el otro pasivo. Descoordinada es una forma que intenta coordinarse sin lograrlo. En la Coordinación Parcial Esporádica hay breves instantes en que se logra la coordinación y luego vuelve a disociarse. En la Coordinación vemos cuerpos que parecen hacer una danza, un movimiento se corresponde con el otro de forma fluida y respetuosa. En la Coordinación Secundaria Esporádica los organismos acuerdan coordinarse no entre ellos sino con un punto fuera de ellos, puede ser una tarea o el propio entrevistador, y lo logran solo a veces. En la Coordinación Secundaria se coordinan muy frecuentemente entre ellos a través de un punto común. En la Disociación Secundaria no hay coordinación ni entre ellos ni con un tercero.

La responsividad parental es la capacidad que tiene la madre de comprender los estados emocionales del hijo y responder eficientemente a ellos. Es una capacidad de apego básica. Va desde No Responde a responder sin involucrarse, como es la Desligada. La Desligada Parcial atiende ciertos requerimientos del hijo, a diferencia de la respuesta de Cuidado y Protección que está atenta a colaborar con el bienestar del hijo sin invadirlo. La Sobreprotección Parcial, por otro lado, es la madre que intenta controlar ciertas áreas del hijo, a veces sus respuestas o su postura, dejando otras libres a su administración. La más controladora es la Sobreprotección Global, donde la madre está pendiente de cada cosa que hace el hijo, sin llegar a la Invasión Protectora que se caracteriza por que la madre coloniza o intenta colonizar todas las áreas de gestión personal del hijo adecuadas a su etapa de desarrollo.

En el control del cuerpo observamos de qué modo el niño logra mover el cuerpo en respuesta al acontecer de la consulta. Es diferente al punto uno de la Tabla donde el niño se presenta con un tono. Aquí apunta a cómo es capaz de variar los movimientos en función de la interacción. Varía desde Escaso Rango De Movimiento, donde el niño está prácticamente bloqueado, a Hipercontrol, donde mantiene una misma postura durante toda la entrevista, así sea que vayan cambiando las temáticas. El Control implica que es capaz de inhibir sus movimientos aun sintiendo el deseo de hacerlo, esto supone que es capaz de evaluar el contexto y la pertinencia de ese movimiento en función de los otros que están ahí. La Flexibilidad se observa en que el niño es capaz de responder y anticiparse a los movimientos del otro, por ejemplo, acerca el lápiz si se da cuenta que el entrevistador lo está buscando. El Control Laxo es un cuerpo que se demora más en reaccionar, pero lo logra. En el Control Simbiótico el cuerpo es capaz de regular sus movimientos solo en función de un otro que lo administra, por ejemplo, la madre lo toca para que no se pare. En el Descontrol el paciente no es capaz de sostener movimientos coherentes con la situación. Puede estar muy inquieto, incluso ser incapaz de estar sentado durante la entrevista.

La dimensión de la teoría de la mente implica alguna elaboración cognitiva, lo que indica mentalización y por lo mismo dimensión social integrada Así, la representación que el niño construye de sí mismo es posible obtenerla desde preguntas sencillas del tipo: ¿cómo te sientes contigo mismo? Cuando hay Desintegración del Sí Mismo, el niño no puede responder coherentemente. Si hay Rechazo se usan palabras como "asco", "odio". En la Descalificación el niño dice: "no me gusto", "soy horrible". Cuando hay Ajuste a la Realidad el niño acepta lo que le parece poco agradable y lo integra con lo que le gusta: "no soy un estrella de cine, pero está bien". En la Muy Valorada el niño se siente muy satisfecho consigo mismo más allá de la imagen corporal. En la Sobrevaloración el niño se valora sin integrar el sobrepeso. En la Hipertrofia Omnipotente el niño parece saltarse el sobrepeso cuando habla de sí mismo, dice que no le importa y tiene argumentos para defenderlo.

La aceptación materna del hijo va desde el Rechazo Total, es decir una madre que no mira y no responde a los requerimientos del hijo, pasando por un Rechazo Parcial, es decir sólo rechaza ciertos aspectos del hijo, la Aceptación Parcial en la que aprueba ciertos aspectos y otros no, y la Aceptación donde a pesar de las diferencias la madre parece respetar al hijo. Otra distinción es la Aceptación Envolvente donde la madre se congracia con el hijo, aunque no tanto como en la Aceptación Cautivante donde la madre seduce al hijo para que esté aliada a ella o más aún en la Rehén donde la madre no sólo seduce sino que bloquea a través de amenazas cualquier acción de autonomía ante una probable exploración del hijo.

En la Negociación observamos de qué forma la diada llega a acuerdos. En un extremo está la Hostilidad, donde ambos parecen tener rabia pero no la manifiestan abiertamente. Es posible deducirla por la rigidez de la cara, los puños apretados. Cuando hay Descalificación aparecen frases dichas uno contra el otro, no se busca el acuerdo sino ganar una lucha de poder. En la Indiferencia se observa que independiente de lo que ocurra no hay manifestaciones emocionales. Cuando hay Respeto se escuchan mutuamente, se ceden los turnos, se miran con tranquilidad. Cuando se negocia con Cariño se perdonan los errores de argumentación, se busca un punto de acuerdo. Si la negociación se centra en la Admiración Mutua, se llega a acuerdos con prontitud. Si el acuerdo es unilateral deberá revisarse para que perdure en el tiempo. En la Idealización ocurrirá una conducta de sumisión que puede no persistir en el tiempo.

En la tabla 3 es posible ver que el riesgo de un PAO se encuentra en cualquiera de los extremos de las dimensiones expuestas. Hacia el lado derecho se encuentra lo que podríamos Ilamar preeminencia del Sistema de alerta (Sistema Simpático) hiperexcitado y el izquierdo la preeminencia del Sistema de alarma sobre compensado con mayor actividad del Sistema Parasimpático. En cualquiera de estos extremos el niño queda 
en riesgo de obesidad si utiliza la ingesta como regulador del estrés. Las díadas que se sitúan más allá del centro $(-8 \mathrm{a}+8)$ tendrían que ser derivadas y tratadas con psicoterapia antes de sugerir intervenciones tradicionales o quirúrgicas, ya que hay alta probabilidad que se reinstale el PAO (tabla 1) y con ello fracase la intervención tradicional.

\section{CONCLUSIONES}

La regulación de la ingesta se encuentra ligada a la regulación del estrés y este a la regulación social (relacional). Comer y amar son dos verbos que se conjugan juntos al inicio de la vida. El Eje HPA se regula a través de múltiples circuitos, uno de ellos es a través del cerebro social, otro más primitivo aún es el SR. Separar el SR de la ingesta así como crear diversos circuitos inhibitorios del HPA son tareas de los procesos de desarrollo mental (mentalización) que se pueden ver afectadas con la interacción biográfica y que requieren reparación a través de cambios en la regulación afectiva social. La modulación eficiente del estrés integra y complejiza diversos circuitos cerebrales durante el desarrollo. Estos cambios se adquieren con mayor facilidad durante la infancia y adolescencia ya que son etapas de integración cerebral acelerada.

Se presenta un modelo y herramientas para medirlo en la práctica clínica. Se propone el PAO como una respuesta maladaptativa establecida tempranamente que liga los sistemas de recompensa a la modulación del eje HPA en ausencia de suficiente regulación social .Posteriores comunicaciones podrían dar cuenta de la utilidad del mismo.

\section{RESUMEN}

La obesidad parece ser una condición compleja. Los tratamientos actuales se basan en modelos simples de entrada y salida de energía con regulares resultados a corto plazo. Es posible que cambiando el modelo de comprensión se logren resultados a más estables en el tiempo. A través de la neurobiología se propone considerar la posible existencia de un patrón

\section{TABLA 3}

Pauta de observación y evaluación clínica de la relación diádica.

\begin{tabular}{|c|c|c|c|c|c|c|c|c|}
\hline & $\begin{array}{l}\text { Dimensión/ } \\
\text { Intensidad en } \\
\text { puntaje }\end{array}$ & $\begin{array}{c}1 \\
(-3)\end{array}$ & $\therefore \begin{array}{c}2 \\
(-2)\end{array}$ & $\begin{array}{c}3 \\
(-1)\end{array}$ & $\begin{array}{c}4 \\
(0)\end{array}$ & $\begin{array}{c}5 \\
(1)\end{array}$ & $\begin{array}{c}6 \\
(2)\end{array}$ & $\begin{array}{c}7 \\
(3)\end{array}$ \\
\hline \multirow{5}{*}{$\begin{array}{l}\text { Derivados } \\
\text { de la } \\
\text { Teoria del } \\
\text { Apego }\end{array}$} & $\begin{array}{l}\text { Postura del } \\
\text { Nino }\end{array}$ & $\begin{array}{l}\text { Derrumbado } \\
\text { Pasivo }\end{array}$ & Laxo & $\begin{array}{l}\text { Atento } \\
\text { Tranquilo }\end{array}$ & $\begin{array}{l}\text { Alerta } \\
\text { Tranquilo }\end{array}$ & Hiperalerta & Amenazado Activo & $\begin{array}{l}\text { Derrumbado } \\
\text { Alerta }\end{array}$ \\
\hline & $\begin{array}{l}\text { Postura de la } \\
\text { Madre }\end{array}$ & Confusa & Pasiva & Tranquila & $\begin{array}{l}\text { Alerta } \\
\text { Tranquila }\end{array}$ & Hiperalerta & $\begin{array}{l}\text { Defensiva } \\
\text { Amenazada }\end{array}$ & Delirante \\
\hline & $\begin{array}{l}\text { Coordinación } \\
\text { Diádica }\end{array}$ & Disociada & Descoordinada & $\begin{array}{l}\text { Coordinación } \\
\text { Parcial } \\
\text { Esporádica }\end{array}$ & Coordinación & $\begin{array}{l}\text { Coordinación } \\
\text { Secundaria } \\
\text { Esporádica }\end{array}$ & $\begin{array}{l}\text { Coordinación } \\
\text { Secundaria }\end{array}$ & $\begin{array}{l}\text { Disociación } \\
\text { Secundaria }\end{array}$ \\
\hline & $\begin{array}{l}\text { Responsividad } \\
\text { Parental }\end{array}$ & No Responde & Desligada & $\begin{array}{l}\text { Desligada } \\
\text { Parcial }\end{array}$ & $\begin{array}{l}\text { Cuidado y } \\
\text { Protección }\end{array}$ & $\begin{array}{l}\text { Sobreprotección } \\
\text { Parcial }\end{array}$ & $\begin{array}{l}\text { Sobreprotección } \\
\text { Global }\end{array}$ & $\begin{array}{l}\text { Invasión } \\
\text { Protectora }\end{array}$ \\
\hline & $\begin{array}{l}\text { Control del } \\
\text { Cuerpo }\end{array}$ & $\begin{array}{l}\text { Control } \\
\text { Simbiótico } \\
\text { laxo }\end{array}$ & $\begin{array}{l}\text { Escaso Rango } \\
\text { De Movimiento }\end{array}$ & Control Laxo & Flexibilidad & Control & Hipercontrol & Descontrol \\
\hline \multirow{3}{*}{$\begin{array}{l}\text { Derivados } \\
\text { de la } \\
\text { Teoría de } \\
\text { la Mente }\end{array}$} & $\begin{array}{l}\text { Representación } \\
\text { del Sí Mismo del } \\
\text { Niño }\end{array}$ & $\begin{array}{l}\text { Desintegración } \\
\text { Del Sí Mismo }\end{array}$ & Rechazo & Descalificación & $\begin{array}{l}\text { Ajuste a la } \\
\text { Realidad }\end{array}$ & Muy Valorada & Sobrevaloración & $\begin{array}{l}\text { Hipertrofia } \\
\text { Omnipotente }\end{array}$ \\
\hline & $\begin{array}{l}\text { Aceptación } \\
\text { Materna del } \\
\text { Niño }\end{array}$ & Rechazo & $\begin{array}{l}\text { Rechazo } \\
\text { Parcial }\end{array}$ & $\begin{array}{l}\text { Aceptación } \\
\text { Parcial }\end{array}$ & Aceptación & $\begin{array}{l}\text { Aceptación } \\
\text { Envolvente }\end{array}$ & $\begin{array}{l}\text { Aceptación } \\
\text { Cautivante }\end{array}$ & Rehén \\
\hline & Negociación & Hostilidad & Descalificación & Indiferencia & Respeto & Cariño & Admiración & Idealización \\
\hline
\end{tabular}

El puntaje medio es 0 . Cualquier puntaje entre $-8 y+8$ está en la franja media. Los puntajes que van más abajo o más arriba son de riesgo. El puntaje se registra con los puntos de la derecha y los de la izquierda por ejemplo: $-6 / 2=4$. Los puntajes de la derecha tienen que ver con la activación del SS (Sistema Simpático) y los de la Izquierda con la activación del SPS (Sistema Parasimpático). Si el puntaje mayor es negativo, la intervención terapéutica debe orientarse a favorecer la distancia del regulador. Si el puntaje mayor es positivo la intervención terapéutica debe orientarse a favorecer la regulación a través de la cercanía del regulador. Si ambos están bajos estamos cerca de la normalidad. Si ambos altos hay riesgo de patología mental más grave.

\begin{tabular}{|c|c|c|c|c|c|c|}
\hline $\mathbf{1}$ & $\mathbf{2}$ & $\mathbf{3}$ & $\mathbf{4}$ & $\mathbf{5}$ & $\mathbf{6}$ & $\mathbf{7}$ \\
$\mathbf{( - 3 )}$ & $\mathbf{( - 2 )}$ & $\mathbf{( - 1 )}$ & $\mathbf{( 0 )}$ & $\mathbf{( 1 )}$ & $\mathbf{( 2 )}$ & $\mathbf{( 3 )}$ \\
\hline-24 & -16 & -8 & 0 & 8 & 16 & 24 \\
\hline
\end{tabular}


adaptativo obesogénico (PAO) donde el funcionamiento del Sistema Nervioso se organizaría tempranamente aumentando la ingesta como principal regulador del estrés. Se propone el PAO como un modelo de comprensión basado en la falla de integración precoz entre los diversos sistemas que regulan el eje HPA. Sería una respuesta maladaptativa establecida tempranamente que liga preferentemente los sistemas de recompensa a la modulación del eje HPA (hipotálamo-pituitaria-adrenal) en ausencia de suficiente regulación social y que podría explicar la obesidad temprana infantil. Se propone una forma para evaluarlo basada en la observación de la relación madre-hijo en la consulta clínica habitual.

Palabras clave: obesidad; estrés; adicción; regulación; peso.

\section{BIBLIOGRAFÍA}

1. Ogden C, Carroll M, Kit B, Flegal K. Prevalence of obesity and trends in body mass index among US children and adolescents, 1999-2010. JAMA 2012; 307(5):483-90.

2. World Health Organization. Obesity and overweight 2014; 311. Available from: http://www.who.int/mediacentre/ factsheets/fs311/en/.

3. Gobierno de Chile. Estrategia Nacional de Salud Para el Cumplimiento de los Objetivos Sanitarios de la Década 2011 - 2020. http://web.minsal.cl/portal/url/item/ c4034eddbc96ca6de0400101640159b8.pdf

4. Atalah E. Epidemiología de la Obesidad en Chile. Rev Med Clin Condes, 2012; 23(2):117-23. http://www. clc.cl/Dev_CLC/media/Imagenes/PDF\%20revista\%20 m\%C3\%A9dica/2012/2\%20marzo/Dr_Atala-3.pdf

5. Mardones F. Obesidad en la niñez en Chile: un tema pendiente. Centro de Políticas Públicas UC. 2010; 5: 41. http://repositorio.uc.cl/xmlui/bitstream/handle/123456789/1758/564096.pdf?sequence $=1$

6. Moraga F, Rebollo MJ, Bórquez P, Cáceres J, Castillo C. Tratamiento de la obesidad infantil: Factores pronósticos asociados a una respuesta favorable. Rev Chil Pediatr 2003; 74: 374-80 http://ref.scielo.org/p42×2v

7. Anderson $\mathrm{GH}$, Catherine NL, Woodend DM, Wolever TM. Inverse association between the effects of carbohydrates and blood glucose and subsequent short-term food intake in young men. Am J Clin Nutr. 2002; 76(5): 1023-30.

8. Schwartz MW, Woods SC, Porte D Jr, Seeley RJ, Baskin $D G$. Central nervous system control of food intake. Nature 2000; 404(6778): 661-71.

9. Metius-Snyder M , Lustig R, Childhood obesity: adrift in the "Limbic Traingle" Ann. Rev.Med. 2008;59:147-62.

10. Dallman MF, Pecorano N, La Fleur SE, Warne JP, Gisberg $A B$, Akana SF, Laugero KC, Houshyar $H$, Strack AM, Bhatnagar S, Bell ME. Glucocorticoids, chronic stress, and obesity. Prog Brain Res. 2006; 153: 75-105.

11. Pecoraro N, Reyes F, Gomez F, Bhargava A, Dallman MF. Chronic stress promotes palatable feeding, which reduces signs of stress: feedforward and feedforward and feedback effects of chronic stress. Endocrinology 2004; 145(8):3754-62.

12. Boyce WT, Ellis BJ. Biological sensitivity to context: I. an evolutionary-developmental theory of the origins and functions of stress reactivity. Dev Psychopathol. 2005; 17(2): 271-301.

13. Hebb DO. Emotion in man and animal: an analysis of the intuitive processes of recognition. Psychol. Rev. 1946; 53:88-106.

14. Schore AN. Attachment and the regulation of the right brain. Attach Hum Dev. 2000 Apr; 2(1):23-47.

15. Panksepp J. A critical role for "affective neuroscience" in resolving what is basic about basic emotions. Psychol Rev. 1992; 99(3):554-60.

16. Agrawal, H., Gunderson, J., Holmes, B.M., \& Lyons-Ruth, $K$. Attachment studies with borderline patients: A review. Harvard Rev Psych, 2004; 12: 94-104.

17. Fosha D. The dyadic regulation of affect. J Clin Psychol. 2001; 57(2):227-42.

18. Fonagy P. Teoría del apego y psicoanálisis. Barcelona, Espaxs, S.A. Publicaciones Médicas, 2001.

19. Cordella P. Actos del cuerpo: semiosis preverbal. Tesis doctoral inédita, Universitá di Bologna, Bologna, 2011.

20. Johnson PL, Truitt $W$, Fitz $S D$, Minick $P E$, Dietrich $A$, Sanghani S, Traskman-Bendz L, Goddard AW, Brundin L, Shekhar A. A key rol for orexin in panic anxiety. Nat. Med. 2010 ; 16(1):111-5. http://www.ncbi.nlm.nih.gov/pmc/ articles/PMC2832844/

21. Jauch-Chara K, Oltmanns KM. Obesity - A neuropsychological disease? Systematic review and neuropsychological model. Prog Neurobiol. 2014; 114: 84-101.

22. Ida T, Nakahara K, Murakami T, Hanada $R$, Nakazato M, Murakami N. Possible involvement of orexin in stress reaction in rats. Biochem Biophys Res Commun. 2000; 270(1):318-23.

23. Zieauddeen H, Farooqi IS, Fletcher PC. Obesity and brain: how convincing is the addiction model? Nat Rev Neurosci. 2012; 13(4): 279-86.

24. Vittoz NM, Berridge CW. Hypocretin/orexin selectively increases dopamine efflux within the prefrontal cortex: involvement of the ventral tegmental area. Neuropsychopharmacol; 31(2): 384-95. http://www.nature.com/npp/ journal/v31/n2/full/1300807a.html

25. Levine AS, Billington CJ. Opioids as agents of rewardrelated feeding: a consideration of the evidence. Physiol Behav. 2004; 82:57-61.

26. Baik JH. Dopamine signaling in reward-related behaviors. Front Neural Circuits. 2013 Oct 11; 7:152. http://www. ncbi.nlm.nih.gov/pmc/articles/PMC3795306/

27. Fogassi L, Ferrari PF, Gesierich B, Rozzi S, Chersi F, Rizzolatti G. Parietal Lobe: from Action Organization to Intention Understanding. Science, 2005 29;308(5722): 662-7.

28. Rizzolatti G, Craighero L. The Mirror-Neuron System. Annu Rev Neurosci. 2004; 27:169-92.

29. Gallese V. Mirror neurons and intentional attunement: commentary on Olds. J Am Psychoanal Assoc. 2006 Winter; 54(1):47-57.

30. Kim JJ, Diamond DM. The stressed hippocampus, synaptic plasticity and lost memories. Nat Rev Neurosci. 2002; 3(6): 453-62.

31. Mahler M. The psychological birth of the human infant: symbiosis and individuation. New York, Basic Books Inc, 1975.

32. Ochs $E$, Shohet $M$. The cultural structuring of mealtime socialization. New Dir Child Adolesc Dev. 2006 ; 111:35-49. http://www.ncbi.nlm.nih.gov/pmc/articles/ PMC2896222/

33. Stein E. Sobre el problema de la empatía. Madrid, Trotta, 2004.

34. Gallese $V$, Keysers $C$, Rizzolatti $G$. A unifying view of the basis of social cognition. Trends Cogn Sci. 2004; 8(9): 396-403.

35. Iacoboni M, Molnar-Szakacs I, Gallese V, Buccino G, Mazziotta JC, Rizzolatti G. Grasping the intentions of others 
with one's own mirror neuron system. PLoS Biol. 2005; 3(3);e79: 529-535. http://www.ncbi.n/m.nih.gov/pmc/ articles/PMC1044835/

36. Del Giudice M, Manera V, Keysers C. Programmed to learn? The ontogeny of mirror neurons. Dev Sci. 200; 12(2): 350-63.

37. Levenson RW, Ekman P. Difficulty does not account for emotion-specific heart rate changes in the directed facial action task. Psychophysiol. 2002; 39(3):397-405.

38. Feldman R. Eidelman Al. Parent-infant synchrony and the socialemotional development of triplets. Dev Psychol. 2004; 40(6):1133-47.

39. Tronick E. Nadel J, Muir D. Why is connection with others so critical? The formation of dyadic states of consciousness and the expansion of individuals' states of consciousness: coherence governed selection and the co-creation of meaning out of messy meaning making. Emotional Development: Recent Research Advances. New York, Oxford, Oxford University Press, 2005, 11.

40. Trevarthen C. First things first: infants make good use of the sympathetic rhythm of imitation, without reason or language. J Child Psychothe. 2005; 31(1): 91-113.

41. Sanders P. La lógica considerada como semiótica. Madrid, Biblioteca Nueva, 2007.

42. Tomasello M, Carpenter M, Call J, Behne T, Moll H. Understanding and sharing intentions: The origins of cultural cognition. Behav Brain Sci. 2005; 28(5): 675-91; discussion 691-735.

43. Perry BD, Pollard R. Homeostasis, stress, trauma, and adaptation. A neurodevelopmental view of childhood trauma. Child Adolesc Psychiatr Clin N Am. 1998; 7(1):33-51.

44. Ainsworth MS, Blehar MC, Water, E, Wall S. Patterns of attachment: A psychological study of the Strange Situation. Hillsdale, Lawrence Erlbaum A. Inc, 1978.

45. Karlen $L R$. The two-person unconscious: Intersubjective dialogue, enactive relational representation, and the emergence of new forms of relational organization. Psych Inq. 1999;19(4): 576-617.

46. Cordella P. Campos de Sintonía Somática Durante el Desarrollo Infantil. Rev Chil Pediatr. 2009;80 (5): 407-19.

47. Caglar-Nazali HP, Corfield F, Cardi V, Ambwani S, Leppanen J, Olabintan O, Deriziotis S, Hadjimichalis A, Scognamiglio P, Eshkevari E, Micali N, Treasure J. A systematic review and meta-analysis for social processes in eating disorders. Neurosci Biobehav Rev. 2014; 42:55-92.

48. Hasler G, Pine DS, Kleinbaum DG, Gamma A, Luckenbaugh $D$, Ajdacic V, Eich D, Rossler W, Angst J. Depressive symptoms during childhood and adult obesity: the Zurich Cohort Study. Mol Psychiatry. 2005; 10(9): 842-50. http://www. nature.com/mp/journal/v10/n9/full/4001671a.html

49. Stice E, Figlewicz DP, Gosnell BA, Levine AS, Pratt WE. The contribution of brain reward circuits to the obesity epidemic. Neurosci Biobehav Rev. 2013. 37(9): 2047-58.

50. Cordella P. Anorexia Bulimia Obesidad. Reflexiones con familias y pacientes. Santiago, Chile, Ediciones UC, 2010.

51. Anderson, GH, LA Catherine N, Woodend DM, Wolevelnverse $T$. Association between the effect of carbohydrates on blood glucose and subsequentshort-term food intake in young men. Am J Clin Nutr. 2002;76(5):1023-30. http:// ajcn.nutrition.org/content/76/5/1023.full.pdf+html

52. Arcelus J, Haslam M, Farrow C, Meyer C. The role of interpersonal functioning in the maintenance of eating psychopathology: a systematic review and testable model. Clin Psychol Rev. 2013; 33(1):156-67.
53. Dallman MF, Pecoraro NC, la Fleur SE. Chronic stress and comfort foods: self-medication and abdominal obesity. Brain Behav Immun. 2005; 19(4):275-80.

54. Berthoud HR, Morrison C. The brain, appetite, and obesity. Annu Rev Psychol. 2008; 59:55-92.

55. Finlayson $G$, King $N$, Blundell JE. Liking vs. wanting food: Importance for human appetite control and weight regulation. Neurosci Biobehav Rev. 2007; 31(7): 987-1002.

56. Corsica JA, Pelchat ML. Food addiction: true or false? Curr Opin Gastroenterol. 2010; 26(2):165-9.

57. Feldman R, Mayes LC, Swain JE. Interaction synchrony and neural circuits contribute to shared intentionality. Behav Brain Sci. 2005; 28(5): 697-698

58. Maroun M. Stress reverses plasticity in the pathway projecting from the ventromedial prefrontal cortex to the basolateral amygdala. Eur J Neurosci. 2006; 24(10):2917-22.

59. Lewis M, Carmody DP. Self-representation and brain development. Dev Psychol. 2008; 44(5): 1329-1334.

60. Lewis M, Ramsay D. Development of Self-recognition, personal pronoun use, and pretend play during the 2nd year. Child Dev. 2004; 75(6):1821-31.

61. DelParigi A, Chen K, Salbe AD, Reiman EM, Tataranni PA. Sensory experience of food and obesity: a positron emission tomography study of the brain regions affected by tasting a liquid meal after a prolonged fast. Neuroimage, 2005; 24(2):436-4

62. Chalew S, Nagel H, Shore S. The hypothalamic-pituitaryadrenal axis in obesity. Obes Res. 1995; 3(4):371-82.

63. Dallman MF, Akana SF, Pecoraro NC, Warne JP, la Fleur $S E$, Foster MT. Glucocorticoids, the etiology of obesity and the metabolic syndrome. Curr Alzheimer Res. 2007; 4(2):199-204.

64. Hruby R, Maas LM, Fedor-Freybergh PG. Early brain development toward shaping of human mind: an integrative psychoneurodevelopmental model in prenatal and perinatal medicine. Neuro Endocrinol Lett. 2013; 34(6):447-63.

65. Taylor GJ. Recent developments in alexithymia theory and research. Can J Psychiatry. 2000; 45(2), 134-42.

66. Darrow SM, Follette WCA. A behavior analytic interpretation of alexithymia. J Contex Behav Sci. 2014 Apr; 3(2):98-108. http://www.sciencedirect.com/science/ journal/22121447/3

67. Trevarthen C, Aitken KJ. Brain development, infant communication, and empathy disorders: Intrinsic factors in child mental health. Dev Psychopathol. 1994; 6(4): 597-633. http://dx.doi.org/10.1017/S0954579400004703

68. Ainsworth MD. Attachments across the lifespan. Bull N Y Acad Med. 1985 Nov; 61(9):792-812. http://www.ncbi. nlm.nih.gov/pmc/articles/PMC1911889/

69. Volkow ND, O'Brien CP. Issues for DSM-V: should obesity be included as a brain disorder? Am J Psychiatr. 2007; 164(5): 708-710. http://ajp.psychiatryonline.org/article. aspx?articleid $=98310$

70. Cordella P. Incluir la obesidad en el manual de enfermedades mentales (DSM-IV)?. Rev Chil Nutr. 2008; 35(3):181-187. http://ref.scielo.org/wdy232

71. Moll H, Tomasello, M. How 14- and 18-month-olds know what others have experienced. Dev Psychol. 2007; 43(2): 309-17.

72. De Niet J, Timman R, Rokx C, Jongejan M, Passchier J, van Den Akker E. Somatic complaints and social competence predict success in childhood overweight treatment. Int $J$ Pediatr Obes. 2011; 6(2-2):e472-9.

73. Russell JA, Bachorowski JA, Fernandez-Dols JM. Facial and 
vocal expressions of emotion. Annu Rev Psychol. 2003; 54:329-49.

74. Cserjési $R$, Vermeulen $N$, Lénárd L, Luminet $O$. Reduced capacity in automatic processing of facial expression in restrictive anorexia nervosa and obesity. Psychiatry Res. 2011; 188(2): 253-7.

75. Hadley W, Stewart A, Hunter HL, Affleck K, Donenberg $G$, Diclemente R, Brown LK. Reliability and validity of the dyadic observed communication scale (DOCS). J Child Fam Stud. 2013; 22(2):279-287. http://www.ncbi.nlm.nih. gov/pmc/articles/PMC3639442/

76. Luyten P, Vliegen N, Van Houdenhove B, Blatt SJ. Equifinality, multifinality, and the rediscovery of the importance of early experiences: pathways from early adversity to psychiatric and (functional) somatic disorders. Psychoanal Study Child. 2008; 63:27-60. 